\title{
The confining transition in the bosonic BMN matrix model
}

\author{
Yuhma Asano, ${ }^{a}$ Samuel Kováčik ${ }^{b}$ and Denjoe O'Connor ${ }^{b}$ \\ ${ }^{a}$ KEK Theory Center, High Energy Accelerator Research Organization, \\ 1-1 Oho, Tsukuba, Ibaraki 305-0801, Japan \\ ${ }^{b}$ School of Theoretical Physics, Dublin Institute for Advanced Studies, \\ 10 Burlington Road, Dublin 4, Ireland \\ E-mail: yuhma@post.kek.jp, skovacik@stp.dias.ie, denjoe@stp.dias.ie
}

ABStRaCt: We study the confining/deconfining phase transition in the mass deformed Yang-Mills matrix model which is obtained by the dimensional reduction of the bosonic sector of the four-dimensional maximally supersymmetric Yang-Mills theory compactified on the three sphere, i.e. the bosonic BMN model. The $1 / D$ (with $D$ the number of matrices) expansion suggests that the model may have two closely separated transitions. However, using a second order lattice formulation of the model we find that for the small value of the mass parameter, $\mu=2$, those two apparent critical temperatures merge at large $N$, leaving only a single weakly first-order phase transition, in agreement with recent numerical results for $\mu=0$ (the bosonic BFSS model).

KeYwords: Lattice Quantum Field Theory, M(atrix) Theories, Gauge-gravity correspondence

ArXiv EPrint: 2001.03749 


\section{Contents}

1 Introduction 1

2 The model and observables $\quad 3$

$3 \quad$ Phase transition(s) of the model with $\mu=2 \quad 5$

4 Conclusions 10

\section{Introduction}

Dimensionally reduced Yang-Mills models provide some of the simplest candidates for understanding gauge/gravity duality and testing the gravitational predictions for gauge theory observables. They arise in a variety of contexts. The supersymmetric versions such as four-dimensional $\mathcal{N}=4$ supersymmetric Yang-Mills theory (SYM) reduced to one dimension, time, known as the BFSS model $[1,2]$ and its maximally supersymmetric mass deformed version known as the BMN model [3] are conjectured to provide non-perturbative definitions of M-theory. Both bosonic and supersymmetric models are matrix quantum mechanical models which also arise as non-commutative deformations of membranes and supermembranes respectively [1,4]. Membranes propagating on trivial backgrounds give rise to BFSS type models [1] while those on non-trivial pp-wave backgrounds [4] give rise to BMN type models. They also describe the dynamics of D0-branes.

When $\mathcal{N}=4 \mathrm{SYM}$ is instead reduced to maximally supersymmetric two-dimensional quantum field theory on $\mathbb{R} \times S^{1}$ and then considered in a thermal bath at very high temperature the fermions decouple and the model reduces to a purely bosonic model which is equivalent to the bosonic BFSS model at a temperature equivalent to the inverse period of the spatial $S^{1}$. The resulting model is the bosonic BFSS model. Alternatively considering the dimensional reduction ${ }^{1}$ of $\mathcal{N}=4 \mathrm{SYM}$ on $\mathbb{R} \times S^{3}$ gives rise to the BMN model. Its bosonic sector is studied in this paper and referred to as the bosonic BMN model. It is also a mass deformation of the bosonic BFSS model and corresponds to a non-commutative deformation of the bosonic membrane propagating on a pp-wave background in eleven dimensions [4].

This family of quantum matrix models has a surprisingly rich phase structure including confining/deconfining phase transitions [5-9] as the temperature is varied.

When the mass parameter, $\mu$, of the model is large the model reduces to a gauged Gaussian model, which is easily solved and has an apparent first-order confining/deconfining phase transition [6, 7]. This transition would continue to smaller $\mu$ and based on the gauge/gravity duality conjecture for two-dimensional maximal SYM it should be connected to the Gregory-Laflamme $[10,11]$ transition in the dual gravitational theory at zero mass deformation.

\footnotetext{
${ }^{1}$ In this truncation higher modes are dropped while preserving maximal supersymmetry.
} 
The massless bosonic BFSS model has received much attention already in previous studies [12-18]. The earliest study was inclined to conclude that there was a single transition [12] but the $1 / D$ expansion [16] suggested the system has in fact two closely separated transitions and this was supported by numerical studies [13] at relatively small $N$. However, more recent studies [15, 17] at larger $N$ and new analytic results [18] find evidence of a single confining/deconfining first-order phase transition. Our study of the BMN model gives the same conclusion that there is only a single transition as $[12,15,17,18]$.

Both the bosonic BMN and BFSS models have also attracted much attention recently $[17,19]$ in the context of partial deconfinement [20-22] where it is argued that these models exhibit deconfinement of some of the degrees of freedom but a subgroup $\mathrm{SU}(M) \subset \mathrm{SU}(N)$ remains confined. This is an intriguing suggestion. However, in these matrix models, such a phase does not seem to appear as a stable phase in the canonical ensemble [17]. Rather what seems to happen is that the transition appears to be a standard first-order one with a rounded critical region at finite $N$ where the system fluctuates between the two phases. In particular the low-temperature phase has the characteristic large fluctuations at finite $N$ of the confined phase near a Hagedorn transition. A more detailed study is warranted in the immediate vicinity of the transition.

The principal results of the current paper are:

- Identification of the transition temperature with precision for $\mu=2$.

- Detection of two pseudo-transition temperatures at finite $N$ that tend to merge in the large- $N$ limit. We bound their difference to $T_{c 2}-T_{c 1}=0.0018(21)$, which is consistent with $T_{c 2}=T_{c 1}$ and therefore a single transition.

- Verification that the model with $\mu=2$ has a single first-order phase transition at $T_{c}=0.915 \pm 0.005$.

- For large $N$ the system fluctuated in the transition region between the approximately uniform phase and the critical gapped phase with eigenvalue density $\rho(\theta)=\frac{1+\cos (\theta)}{2 \pi}$.

Our results for $\mu=2$ should be close to those of $\mu=0$ where the transition has an interpretation in terms of black strings/black hole transitions if gauge/gravity duality holds. With this interpretation our results are in accord with the conclusion of [23] where non-uniform black strings are found to be unstable in lower dimensions and in particular for the gravity dual of the bosonic BFSS model. The thermodynamics we find is also in accord with other studies [24-26].

Furthermore support for our results comes from $\mathcal{N}=4 \mathrm{SYM}$ in the large- $N$ limit on $S^{3} \times S^{1}$, where the partition function depends only on the ratio of the radii $R$ of $S^{3}$ and $\beta$ of $S^{1}$, i.e. $\beta / R$, and the 't Hooft coupling. Witten argued [27] that the model has a confining/deconfining transition dual to the Hawking-Page transition in the dual gravitational theory. If this transition occurs at sufficiently high temperature then the fermions will decouple and one would expect that the resulting transition would be smoothly connected to that of the bosonic BMN and BFSS models. Our results are in accord with this picture. 
The paper is organised as follows: in section 2 we briefly review the model, define our notation and list the observables we consider. Section 3 gives our main results and we finish with conclusions and comments in 4 .

\section{The model and observables}

The BMN matrix model is the quantum mechanical matrix model obtained from a noncommutative deformation of the relativistic supersymmetric membrane, with Nambu-Goto action in lightcone coordinates propagating in eleven-dimensional spacetime on a pp-wave background [3, 4]. The bosonic BMN model is the corresponding bosonic model, i.e. the BMN model without fermions. More precisely, the model is a quantum matrix model, with $\mathrm{SU}(N)$ gauge symmetry, consisting of 9 Hermitian $N \times N$ matrices whose Euclidean finite temperature action is given by

$$
\begin{aligned}
S[X, A]=N \int_{0}^{\beta} d \tau \operatorname{Tr}[ & \frac{1}{2} D_{\tau} X^{i} D_{\tau} X^{i}-\frac{1}{4}\left(\left[X^{r}, X^{s}\right]+\frac{i \mu}{3} \varepsilon^{r s t} X_{t}\right)^{2} \\
& \left.-\frac{1}{2}\left[X^{r}, X^{m}\right]^{2}-\frac{1}{4}\left[X^{m}, X^{n}\right]^{2}+\frac{1}{2}\left(\frac{\mu}{6}\right)^{2} X_{m}^{2}\right],
\end{aligned}
$$

where $i=1, \cdots, 9, r, s=1,2,3$ and $m, n=4, \cdots, 9$. Also, $\beta=1 / T$ is the inverse temperature, $\mu$ is the mass parameter and $D_{\tau} \cdot=\partial_{\tau} \cdot-i[A, \cdot]$ is the covariant derivative. The $\mathrm{SO}(9)$ symmetry is explicitly broken to $\mathrm{SO}(6) \times \mathrm{SO}(3)$ by the mass terms and the cubic Myers term.

Since we are interested in non-perturbative results for the model, we investigate the model numerically using the hybrid Monte Carlo algorithm described in [14]. We use the lattice formulation of the model where the matrices $X^{i}$ are placed on lattice sites and the gauge field $A$ on links. The Euclidean time variable $\tau$ is discretised as $\tau \rightarrow \beta k / \Lambda$, where $k=1, \cdots, \Lambda$. We use the second order discretisation of the kinetic term discussed in $[9,28]$ where the quartic term in the momentum expansion of the lattice Laplacian is set to zero. Without loss of generality the coupling constant has been fixed to 1 and all dimensionful quantities are expressed in these natural units.

The lattice model depends on four parameters: $\mu, \beta, \Lambda$ and $N$, the last two of which are to be sent to infinity to obtain the continuum, large- $N$ limit.

Mean values of an observable $\mathcal{O}$ are defined by integration over the ten (or more generally $D+1$ ) Hermitian matrices $X^{r}, X^{m}, A$ via

$$
\langle\mathcal{O}\rangle=\frac{\int[d X][d A] \mathcal{O} e^{-S[X, A]}}{Z}, Z=\int[d X][d A] e^{-S[X, A]} .
$$

In practice, the gauge field $A$ is fixed to be diagonal and time independent with a consequent Vandermonde determinant in the measure as discussed in [14].

We measure the standard set of observables: the energy $E$, the specific heat $C_{\mathrm{v}}$, the 'extent' observable $\left\langle R^{2}\right\rangle$ and the Polyakov loop $\langle|P|\rangle$ which serves as an order parameter in the confining/deconfining transition. We also measure the Myers observable, $M$, which is 
crucial in the full supersymmetric model where, at low temperatures, the fermionic terms stabilise three of the matrices into fuzzy sphere configurations [9], but as discussed below we find no such stable fuzzy sphere configurations for the bosonic model.

Our principal observables are defined as

$$
\begin{aligned}
E & =\frac{1}{N^{2}}\left(-\partial_{\beta}\right) \log Z=\frac{1}{N^{2}}\left\langle\mathcal{O}_{E}\right\rangle, \\
C_{\mathrm{v}} & =\frac{\beta^{2}}{N^{2}} \partial_{\beta}^{2} \log Z=\frac{\beta^{2}}{N^{2}}\left\langle\left(\mathcal{O}_{E}-\left\langle\mathcal{O}_{E}\right\rangle\right)^{2}-\mathcal{O}_{E}^{\prime}\right\rangle, \\
\langle|P|\rangle & =\left\langle\frac{1}{N}|\operatorname{Tr}(\exp (i \beta A))|\right\rangle, \\
\left\langle R^{2}\right\rangle & =\left\langle\frac{1}{N \beta} \int_{0}^{\beta} d \tau \operatorname{Tr}\left(X^{i} X^{i}\right)\right\rangle, \\
M & =\left\langle\frac{i}{3 N \beta} \int_{0}^{\beta} d \tau \varepsilon^{r s t} \operatorname{Tr}\left(X^{r} X^{s} X^{t}\right)\right\rangle,
\end{aligned}
$$

where

$$
\mathcal{O}_{E}=\frac{N}{\beta} \int_{0}^{\beta} d \tau \operatorname{Tr}\left(-\frac{3}{4}\left[X^{i}, X^{j}\right]^{2}-\frac{5}{6} i \varepsilon_{r s t} X^{r} X^{s} X^{t}+2\left(\frac{\mu}{3}\right)^{2} X^{r} X^{r}+2\left(\frac{\mu}{6}\right)^{2} X^{m} X^{m}\right),
$$

and

$$
\mathcal{O}^{\prime}{ }_{E}=\frac{N}{\beta^{2}} \int_{0}^{\beta} d \tau \operatorname{Tr}\left(-\frac{3}{2}\left[X^{i}, X^{j}\right]^{2}-\frac{5}{4} i \varepsilon_{r s t} X^{r} X^{s} X^{t}+2\left(\frac{\mu}{3}\right)^{2} X^{r} X^{r}+2\left(\frac{\mu}{6}\right)^{2} X^{m} X^{m}\right) .
$$

Note: as can be seen from the presence of $\mathcal{O}^{\prime}{ }_{E}$ in the path integral version of the specific heat $C_{\mathrm{v}}$, the propability distribution of $E$ as measured in the path integral does not directly give the probability distribution of the quantum mechanical energy.

There are two additional observables that increase the precision of critical temperature estimates, they will be introduced shortly.

When the mass parameter $\mu$ is very large, the model reduces to a solvable (gauged Gaussian) model. A straightforward calculation $[6,7,29]$ shows that, in the large- $N$ limit, there is a single phase transition with critical temperature $T_{c}=\frac{\mu}{6 \log (3+2 \sqrt{3})}$ where $\langle|P|\rangle$ jumps from $\langle|P|\rangle=0$ to $\langle|P|\rangle=1 / 2$ and then gradually increases to $\langle|P|\rangle=1$ as the temperature is further increased.

For the case with $\mu=0$, known as the bosonic BFSS model, there are already several studies in the literature. These include a perturbative expansion in $1 / D[14,16,30]$, where $D$ is the number of matrices ( $D=9$ in our case), and numerical studies $[13-15,17]$. Both the $1 / D$ expansion [16] and earlier studies [13] reported two closely separated critical tem- 
peratures. The $1 / D$ expansion predicts ${ }^{2} T_{c 1}=0.895 \pm 0.004$ and $T_{c 2}=0.911 \pm 0.002$ with the difference in critical temperatures decreasing with increasing $D$ as $\frac{1}{6 D^{2 / 3} \ln D}$. Between the two critical temperatures, the $1 / D$ expansion predicts that $\langle|P|\rangle$ should gradually increase from 0 to $1 / 2$ with increasing temperature. However, it is conceivable that further increasing the order in the perturbative loop expansion will close the gap resulting in its disappearance in a non-perturbative calculation and hence show that the model has in fact a single transition.

Early numerical studies found reasonable agreement with the one-loop $1 / D$ predictions; however, a refined recent study [17] finds only one transition. They [17] find the single transition occurs between the predicted transitions of the $1 / D$ expansion and when we perform an extrapolation using their figure 7 we estimate $T_{c}=0.89 \pm 0.01$. As we will see below, our analysis of the mass deformed model with $\mu=2$ will agree with the conclusion that there is only one transition for $D=9$.

The $1 / D$ expansion can be easily extended to the mass deformed, bosonic BMN model, in a double expansion in $1 / D$ and perturbation theory in the cubic Myers term. Perturbation in the Myers term can be justified by its small value shown in the figure 1. As argued in [17], it appears that $D=9$ is not sufficiently large to trust the $1 / D$ expansion in predicting the phase transition structure though it gives a reliable indication of the critical region. Our analysis supports this conclusion also for non-zero $\mu$.

\section{Phase transition(s) of the model with $\mu=2$}

In this paper we restrict our study to a single value of $\mu$. We chose $\mu=2$ as the most interesting value, since it is neither large (the asymptotically large mass region of the phase diagram can be accessed analytically), nor small, being of order 1 in natural units for the model.

Figure 1 shows the temperature dependence of the internal energy, $E$, its specific heat $C_{\mathrm{v}},\left\langle R^{2}\right\rangle$ and the Myers term for $\mu=2, \Lambda=24$ and $N=32$ (all with jackknife error bars). Figure 2 shows the temperature dependence and evolution of the Polyakov loop $\langle|P|\rangle$ with increasing $N$. The right panel of figure 2 shows that the peak of the specific heat grows quadratically ${ }^{3}$ with $N$. Due to the rapid increase in the number of degrees of freedom with $N$, the simulations become more demanding and it becomes increasingly difficult to get sufficient data to keep the errors down, especially in the critical region. From the figure it is clear that the system undergoes one or more phase transitions in the vicinity of $T_{c} \sim 0.91$, yet in figure 2 it is difficult to resolve two close transitions as expected from the $1 / D$ expansion. To do so, we analyse the transition(s) in more detail below.

In our choice of gauge fixing [14], where the gauge field is diagonal and placed on the final link, i.e. between $\Lambda$ and 1, the gauge field is fully described by a vector of an-

\footnotetext{
${ }^{2}$ The expression (4.30) of [16] leads to $T_{c 1}=1 / \beta_{c 1}(9)=0.895$; however, inverting $\beta_{c 1}(D)$ and expanding it in $1 / D$ yields $T_{c 1}=0.891$. The same goes for the second critical temperature, obtained as $T_{c 2}=$ $1 / \beta_{c 2}(9)=0.911$ from $\beta_{c 2}(D)=\beta_{c 1}(D)-\frac{\ln D}{6 D^{4 / 3}}$ with the error being 0.002 .

${ }^{3} \mathrm{~A}$ similar quadratic growth was observed for the Polyakov loop susceptibility in [15].
} 

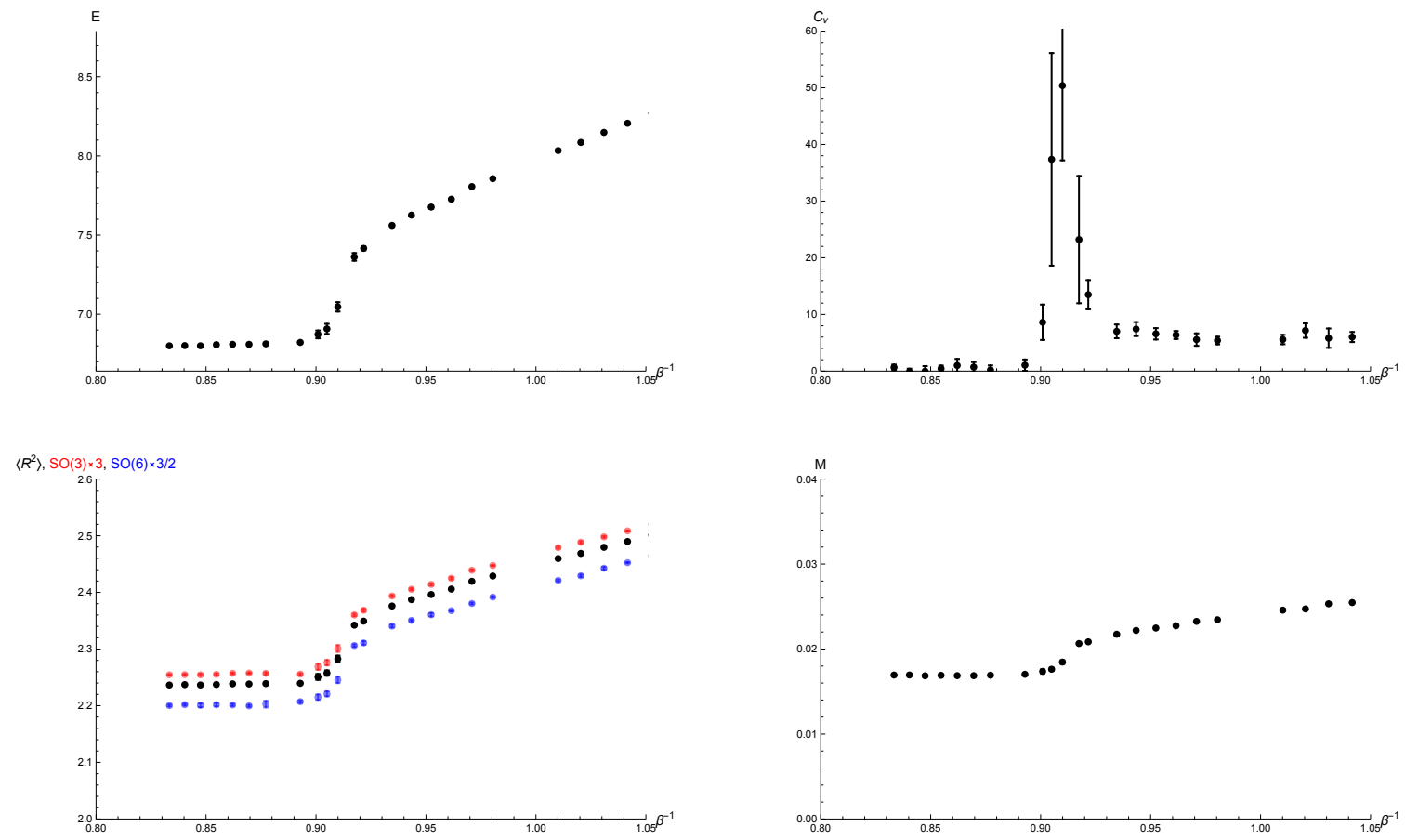

Figure 1. The energy $E$, specific heat $C_{\mathrm{v}},\left\langle R^{2}\right\rangle$ with its rescaled $\mathrm{SO}(3)$ and $\mathrm{SO}(6)$ components and the Myers observables of the model for $\mu=2, N=32$ and $\Lambda=24$ are shown. The Myers observable copies the shape of $\left\langle R^{2}\right\rangle$ (and its $\mathrm{SO}(3)$ and $\mathrm{SO}(6)$ components) but has minuscule expectation values in comparison. All observables point to either a single or multiple transitions around $T \approx 0.91$.

$\mathrm{N}=12,24,32,48$
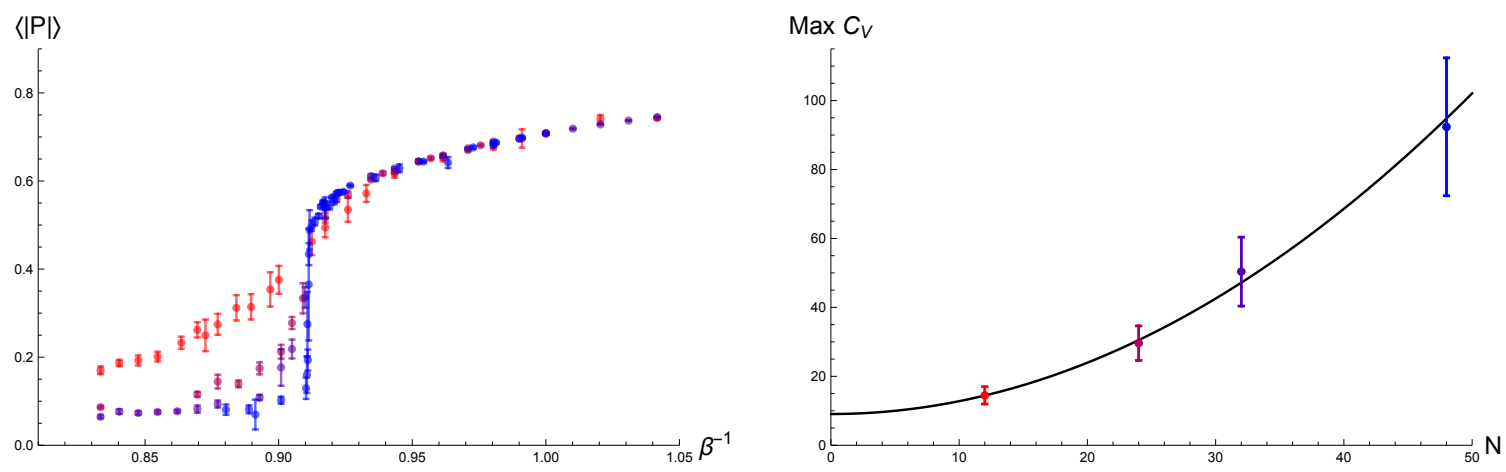

Figure 2. The evolution of the Polyakov loop $\langle|P|\rangle$ for $\mu=2, \Lambda=24$ with increasing $N$. The transition region becomes sharper with larger $N$ but also, due to more demanding simulations, the statistical errors grow. The right figure shows the maximum specific heat against $N$ with fit $C_{\mathrm{v}}^{\mathrm{Max}}=9.1(8)+0.037(2) N^{2}$, adding a linear term increases errors and does not improve the fit. 
gles $-\pi<\theta_{i} \leq \pi$ for $i=1, \ldots, N$. These are described by a probability distribution $\rho_{N}(\theta)=\frac{1}{N} \sum_{i=1}^{N}\left\langle\delta\left(\theta-\theta_{i}\right)\right\rangle$, which in the large- $N$ limit gives distribution $\rho(\theta)$.

True phase transitions occur only in the large- $N$ limit and they are rounded at finite $N$. In the bosonic BMN model there are three distinguishable apparent phases for finite values of $N$. When the temperatures are very low, the distribution is approximately uniform (up to finite- $N$ corrections). As the temperature is increased, the distribution becomes more non-uniform and then it develops a gap. We will sometimes abuse terminology by referring to such transition temperatures as critical temperatures though more strictly they are pseudo, apparent or effective critical temperatures. This should not cause confusion as in the end we will only identify one true critical temperature.

Since the distribution is restricted to the periodic interval $(-\pi, \pi]$ it is convenient to use the Fourier transformation so that $u_{n}$ is defined as the non-trivial $n$-th moment of $\rho(\theta)$,

$$
u_{n}=\int_{-\pi}^{\pi} \rho(\theta) e^{i n \theta} d \theta
$$

Therefore, we also define the following analogous generalizations of the Polyakov loop as ${ }^{4}$

$$
\left\langle\left|P_{n}\right|\right\rangle=\left\langle\frac{1}{N}|\operatorname{Tr}(\exp (i n \beta A))|\right\rangle .
$$

Notice that the first moment $u_{1}$ is actually equal to the expectation value of the Polyakov loop i.e. $u_{1}=\langle|P|\rangle$. The effective potential for $u_{1}$ can be obtained, at least approximately, from the $1 / D$ expansion [16]. It can be expanded as a polynomial with $D$ and temperature dependent coefficients. It is minimised by $u_{1}=0$ for $T<T_{c 1}$, then grows until it reaches $u_{1}=1 / 2$. The prediction of the $1 / D$ analysis is therefore that, as the temperature is increased, beyond the first critical temperature, $T_{c 1}$, the first moment (Polyakov loop) develops a positive expectation value. As the temperature is further increased $\langle|P|\rangle$ grows reaching $\langle|P|\rangle=1 / 2$ at the second critical temperature $T_{c 2}$. Above this second critical temperature the distribution becomes gapped and the second and higher moments become non-zero.

Note that the effective potential in the large- $\mu$ limit is a quadratic function and as the quadratic coefficient flips sign at $T_{c 1}$, the first moment $u_{1}$ jumps immediately to $1 / 2$. Therefore, $T_{c 1}=T_{c 2}$ in this limit and there is a single critical temperature.

For generic $\mu$ at one loop the $1 / D$ expansion predicts two transitions; the first occurring when the Polyakov loop departs from zero and the second when it reaches $1 / 2$, where a Gross-Witten type transition occurs. However, since these transitions are so close and the $\mu=0$ study indicates there is only one transition, it is possible that there are two transitions for non-zero $\mu$ which merge into a single transition at $\mu=0$. Alternatively, there may be a single transition for a range or possibly all values of $\mu$. In an effort to resolve this issue we resort to a non-perturbative lattice study of the model.

\footnotetext{
${ }^{4}$ The $\mathrm{U}(1)$ transformation of the model where $A \rightarrow A+\alpha \mathbf{1}$ is used to remove the phase and one is left with the modulus.
} 


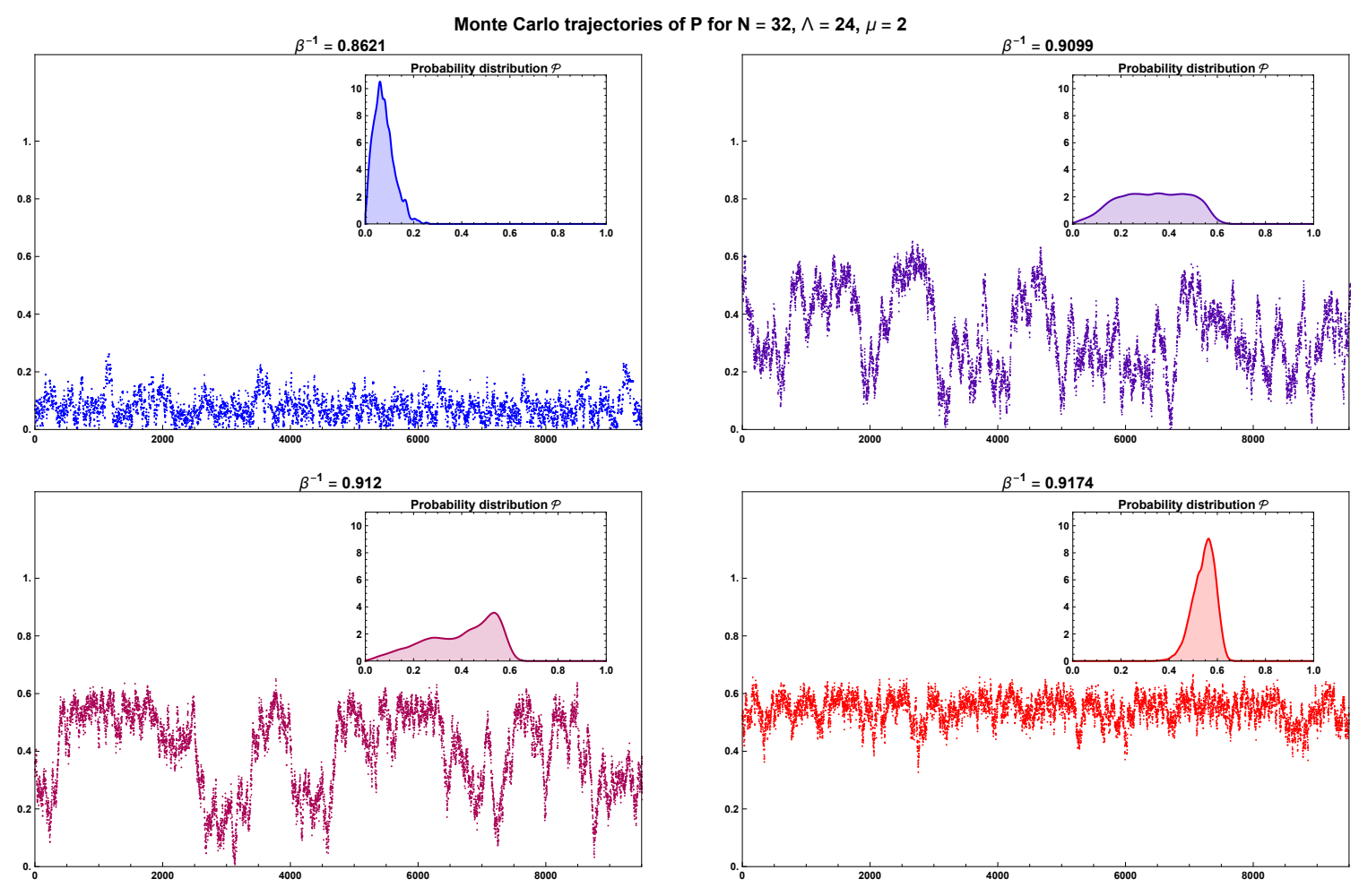

Figure 3. Monte Carlo trajectories of the Polyakov loop, $\langle|P|\rangle$, for $\mu=2, N=32$ and $\Lambda=24$. There is one clear top level and one less visible bottom level. As the temperature is increased, the system tends to spend more Monte Carlo steps in the upper one. The colours are matched with the coloured points in figure 4 . We can observe that the transition is weakly first-order.

To measure the first critical temperature, we need to understand the behaviour around this transition. Monte Carlo trajectories of $\langle|P|\rangle$ are shown in figure 3. From the figure it seems plausible that there are two distinct levels, suggesting a first-order phase transition. This behaviour becomes more articulate at higher $N$. There seems to be one clear level around $\langle|P|\rangle=\frac{1}{2}$ and one significantly below it. Therefore, we define a new observable, $\mathbb{P}$, defined via

$$
\mathbb{P}=\mathbb{P}_{\frac{1}{2}}, \quad \text { with } \quad \mathbb{P}_{x}=\int_{x}^{1} \mathcal{P}(q) d q,
$$

where $\mathcal{P}(q)$ is the probability distribution for the Polyakov loop.

Well below the transition $\mathbb{P}$ is zero, while well above it is one and it grows very quickly around the critical temperature, as seen in the left panel of figure 4. It is closely related to $\langle|P|\rangle$ but seems to be less prone to finite- $N$ effects. The value of $x$ in $\mathbb{P}_{x}$ should be chosen between the value of $\langle|P|\rangle$ in the low-temperature phase $(\sim O(1 / N))$ and the hightemperature phase $(\approx 0.5)$; in fact, we observed that $\mathbb{P}_{0.4}$ and $\mathbb{P}_{0.3}$ gave consistent results to $\mathbb{P}$. We fit the transition region of this curve for $\mathbb{P}$ with a linear function and find its intercept with the temperature axis to obtain $T_{c 1}$. 

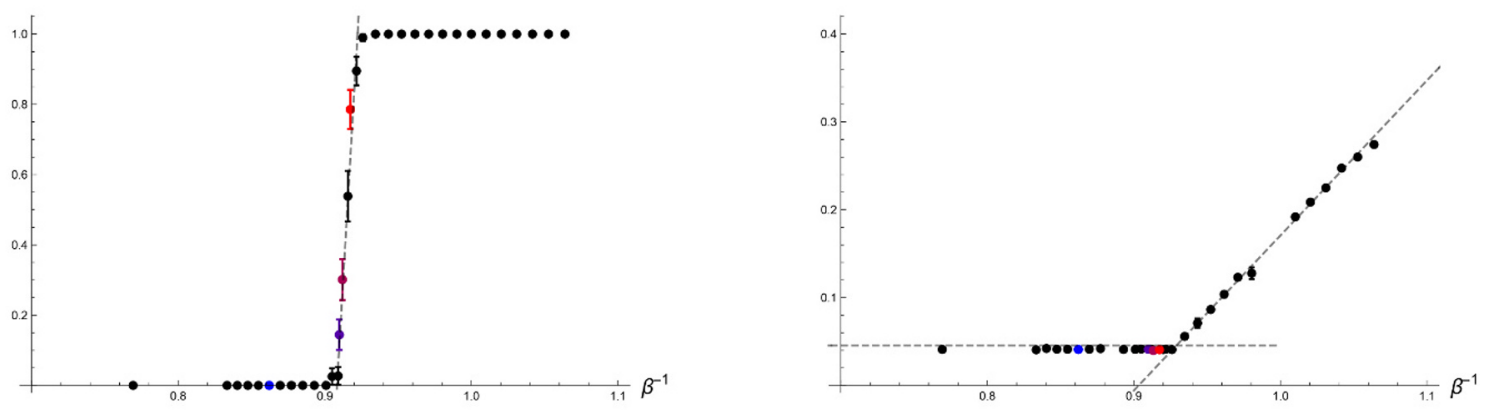

Figure 4. Values of $\mathbb{P}$ and $\left\langle\left|P_{2}\right|\right\rangle$ for $\mu=2, N=32$ and $\Lambda=24$ and increasing value of temperature $T=\beta^{-1}$. The points in the transition region in the left plot were fit by a linear function whose slope increases with increasing $N$. The four coloured points are colour-matched with the trajectories in figure 3 .
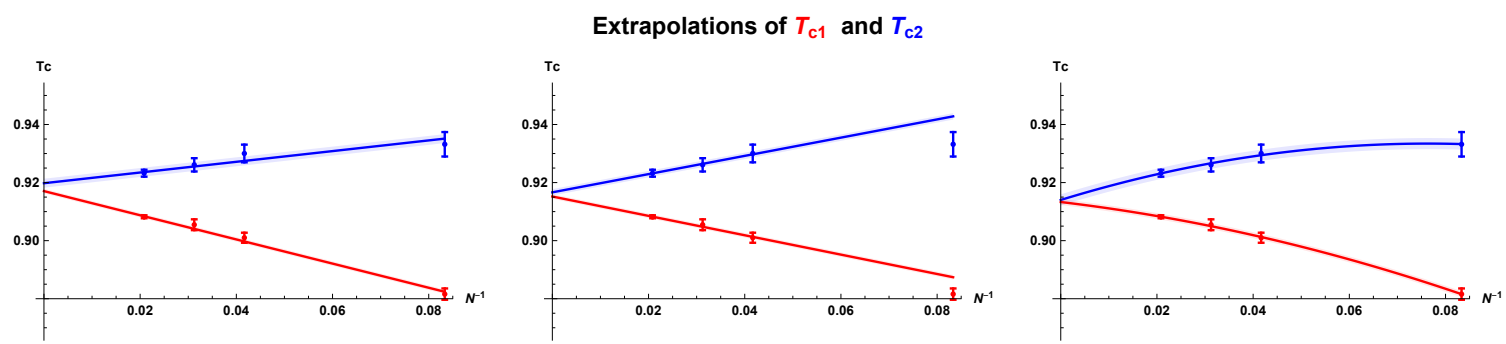

Figure 5. Extrapolations of the critical temperatures from results obtained from $N=12,24,32,48$ with $\Lambda=24$. The left, middle and right plots use a linear fit with all data, a linear fit but with the $N=12$ data omitted, and a quadratic fit, respectively. All fits are functions of $N^{-1}$.

As discussed above, the second moment, $\left\langle\left|P_{2}\right|\right\rangle$, provides a strong marker for a gappedto-ungapped transition. ${ }^{5}$ Higher moments would provide similar information but with larger errors. We present $\left\langle\left|P_{2}\right|\right\rangle$ versus temperature for $\mu=2, N=32$ and $\Lambda=24$ in the right panel of figure 4 . To identify the effective critical temperature from $\left\langle\left|P_{2}\right|\right\rangle$ we fit the data above and below the lower effective critical temperature with two linear functions. All of the data in the low-temperature phase is well fit by a line, and approximately twenty of the data points nearest to the transition are fit by a line in the higher temperature region. The intercept of these two linear fits is then taken as the effective critical temperature (the error is the propagated error of the fitting functions).

We repeated this study with $N=12,24,32,48$ and $\Lambda=24$. Our extrapolation of the results to large $N$ is shown in figure 5 . The results depend only slightly on the form of extrapolation function used as shown in table 1. If one insists that there are indeed two transitions rather than one, the maximum separation of these two transitions must be less than $T_{c 2}-T_{c 1}=0.0018(21)$. However, our numerical simulations strongly suggest that in fact $T_{c 2}-T_{c 1}=0$. We conclude that, in the large- $N$ limit, there is a single transition as in the Gaussian model for large $\mu$.

\footnotetext{
${ }^{5}\left\langle\left|P_{2}\right|\right\rangle$ and higher moments were also discussed in [12] and [15].
} 


\begin{tabular}{|l|c|c|c|}
\hline & Linear & Linear without $N=12$ & Quadratic \\
\hline$T_{c 1}$ & $0.9172(7)$ & $0.9154(7)$ & $0.9137(9)$ \\
\hline$T_{c 2}$ & $0.919(2)$ & $0.917(1)$ & $0.914(2)$ \\
\hline
\end{tabular}

Table 1. Extrapolation of $T_{c 1}$ and $T_{c 2}$ to $N=\infty$ obtained from the fitting functions shown in figure 5 .

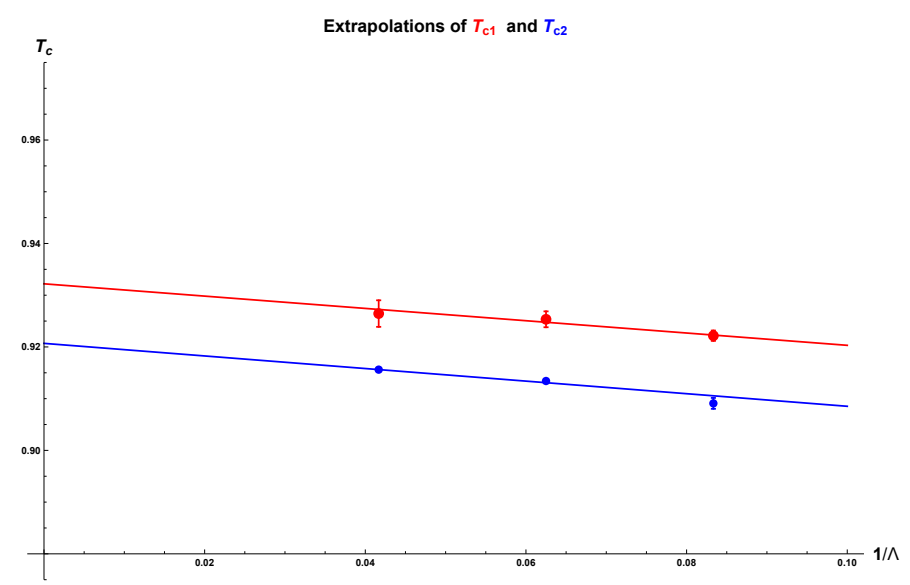

Figure 6. Lattice dependence for $N=32$.

For $N=32$ extrapolation of the effective critical temperature to the continuum limit was performed using $\Lambda=12,16,24$. The measured critical temperatures are well fit in figure 6 with

$$
T_{c 1}(\Lambda)=T_{c 1}(\infty)-\frac{0.12(2)}{\Lambda} \quad \text { and } \quad T_{c 2}(\Lambda)=T_{c 2}(\infty)-\frac{0.12(3)}{\Lambda} .
$$

The gap between the effective critical temperatures was found to scale as

$$
\left(T_{c 2}-T_{c 1}\right)(\Lambda)=\left(T_{c 2}-T_{c 1}\right)(\infty)+\frac{0.003(24)}{\Lambda} .
$$

For $\Lambda=24$ we see that $\left(T_{c 2}-T_{c 1}\right)(24)-\left(T_{c 2}-T_{c 1}\right)(\infty)=0.0001(10)$ and therefore using $\Lambda=24$ seems sufficient so that lattice effects are within the errors of the simulation.

Our conclusion is therefore that in the large- $N$ and continuum limit, for $\mu=2$, the gap between the effective finite- $N$ critical temperatures vanishes and there is a single, uniformto-gapped, first-order phase transition. From the table above and including lattice errors we estimate that the true critical temperature of the continuum, large- $N$, mass deformed model with $\mu=2$ is $T_{c}=0.915 \pm 0.005$.

\section{Conclusions}

In this paper we find that the bosonic BMN model at $\mu=2$ undergoes a single confining/deconfining phase transition in the large- $N$ limit. Combining this result with the result at asymptotically large $\mu$ and the $\mu=0$ study in [17] we conclude that the most 
probable scenario is that there is a single first-order transition with a $\mu$ dependent critical temperature for any value of the mass parameter.

In contrast to this, the supersymmetric version of the model studied in [9] has a rich phase structure ${ }^{6}$ and in addition to the confining/deconfining transition (it has not been established whether there is more than one closely separated transitions for small $\mu$ ), there also is a Myers phase transition, where the Myers observable, (2.3), grows rapidly as the model develops fuzzy sphere backgrounds. No such transition occurs in the bosonic case as seen from the Myers term in figure 1 and the fact that both the $\mathrm{SO}(3)$ and $\mathrm{SO}(6)$ sums of $\left\langle X_{i}^{2}\right\rangle$, contributing to $\left\langle R^{2}\right\rangle$, behave similarly. The absence of fuzzy spheres is not surprising since the $\mathrm{SO}(3)$ sector of the potential in the bosonic model (2.1) is a complete square and hence bounded below by zero ${ }^{7}$ and the zero point fluctuations add an effective positive quadratic contribution which makes the trivial configuration the only stable vacuum. In the supersymmetric model the fermions cancel the bosonic zero point energy. It is therefore the fermions that drive the supersymmetric model into a fuzzy sphere phase and they are required to support the fuzzy sphere background.

We also find in our study, that the eigenvalue distribution for the gauge field in the temperature range $T_{c 1}<T<T_{c 2}$ (where $T_{c 1}$ and $T_{c 2}$ are the pseudo-critical temperatures measured at finite $N$ ) can be fit by the proposed function

$$
\rho=\frac{1}{2 \pi}(1+p \cos \theta), \quad 0<p<1 .
$$

In this case the prediction for the Polyakov loop, or $u_{1}$ of (3.1), in this temperature interval is $u_{1}=\frac{p}{2}$.

This distribution (4.1) does not simply mean the system is in the ungapped, nonuniform phase, which is conjectured to be a partially deconfined phase. The distribution can be realised in simulations via two ways: one way is that the eigenvalue distribution is in this form at each Monte Carlo step, and the other is that this form is realised only as an average of different kinds of distributions over Monte Carlo time. We observe, in this matrix model, the distribution near the transition temperature is realised in the latter way. By examining the eigenvalues in detail we find that what happens is that the eigenvalues fluctuate between the gapped and ungapped phase (see figure 7 and similar behaviour for $\mu=0$ in [17]) and that it is the spacing of the eigenvalues and hence their distribution that changes. Since Monte Carlo simulations should realise possible physical states of the system, superposition of the two phases is how the distribution (4.1) is realised. Therefore it is understood as the interpolation $\rho=(1-p) \rho_{u}+p \rho_{c}$ between the uniform distribution $\rho_{u}=\frac{1}{2 \pi}$ and the critical density $\rho_{c}=\frac{1+\cos (\theta)}{2 \pi}$, where $p$ is the probability of finding the system in the phase with critical density $\rho_{c}$, which corresponds to the endpoint density of the deconfined phase. Although configurations with $0<p<1 / 2$ in the Monte Carlo trajectories, they make a negligible contribution to the distribution.

Note here that, although physical states are realised in simulations, measurement of an observable does not necessarily reproduce the real probability distribution at finite $N$.

\footnotetext{
${ }^{6}$ Its supergravity dual was studied in [33].

${ }^{7}$ In the matrix model of this sector studied in $[31,32,34]$ the transition only occurs for sufficiently negative quadratic term.
} 

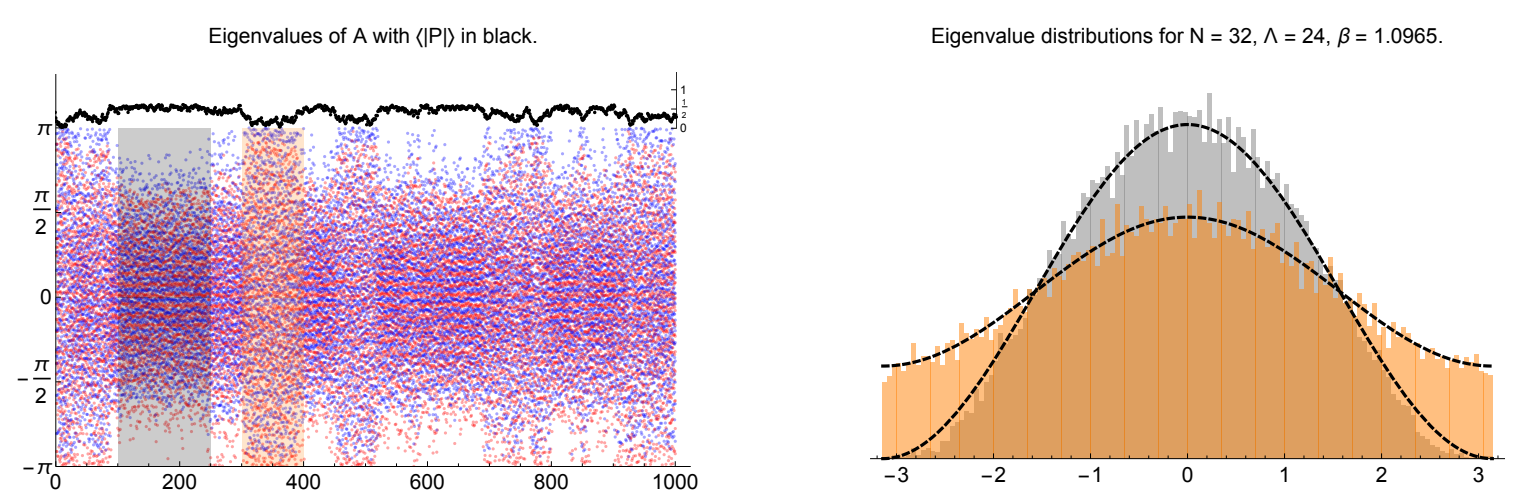

Figure 7. The left figure shows the Monte Carlo evolution (every 10th step is shown) of the eigenvalues together with the evolution of the Polyakov loop $\langle|P|\rangle$. On the right we plot eigenvalue distributions corresponding to eigenvalues on the left. The grey plot shows the distribution evaluated from configurations between MC times 1000 and 2500 corresponding to the Polyakov loop in the upper level around 0.5 while the orange plot shows the distribution between MC times 3000 and 4000 corresponding to the Polyakov loop in the lower level. The system is fluctuating between the approximately uniform distribution $\rho(\theta)=\frac{1+2\langle|P|\rangle \cos (\theta)}{2 \pi}$ with $\langle|P|\rangle=0.22$ and the critical distribution $\rho(\theta)=\frac{1+\cos (\theta)}{2 \pi}$, represented by the dashed curves for the orange and grey histograms in the figure, respectively. For the orange data segment $\langle|P|\rangle=0.22 \pm 0.03$ and for the grey data $\langle|P|\rangle=0.53 \pm 0.01$.

For example, the probability distribution of the energy observable in (2.3) or any other simple observables of energy does not give the quantum mechanical distribution of energy. As noted in section 2, one can see from the specific heat expression in (2.3) that they can be quite different.

Our study suggests that the confining/deconfining transition is a relatively standard weakly first-order one. As expected in such a transition, at finite but large enough $N$, we observe that the peak of the specific heat grows as $N^{2}$ (the number of degrees of freedom) shown in figure 2 and that the two-level features are clearly visible ${ }^{8}$ in figure 3 . That the transition is first-order is also in accord with the findings from the bosonic BFSS model $[15,17]$.

The behaviour of the specific heat in the standard first-order transition is well understood in statistical physics. The renormalization-group approach [35] and other work [36, 37 predict that the transition is smeared over a region whose width goes to zero as the inverse of the number of degrees of freedom and the specific heat has a peak with maximum which grows in proportion to the number of degrees of freedom. This peak for the finite system replaces the discontinuity of the specific heat in the thermodynamic limit. The effect is easily modeled by a partition function built from two extensive Gaussian distributions [38] — one around each peak.

As discussed above, the two-level features are interpreted as a mixture of the two phases in the transition region. The growth of the Polyakov loop, between the two transitions, is

\footnotetext{
${ }^{8}$ We also observed that the two-level feature of the transition becomes clearly visible only above $N \approx 30$, though the growth in the peak of the specific heat in figure 2 is apparent for smaller $N$.
} 
related to the relative amount of time the simulation spends in the upper and lower levels seen in figure 3. The parameter $p$ in (4.1) is then measured by the fraction of Monte Carlo time the system spends in the level $|P| \sim 0.5$ and is closely related to our observable $\mathbb{P}$. In the transition region $\mathbb{P}$ is surprisingly linear as seen in figure 4 . That the slope increases with increasing $N$ is inferred from the convergence of the two pseudo-critical temperatures shown in figure 5 . The linear behaviour of $\mathbb{P}$ in the transition region is probably due to the exceptionally small temperature range over which the finite- $N$ transition occurs.

These finite- $N$ behaviours, such as the interpolation of two kinds of distributions in (4.1) and the $N$-dependence of the specific heat, can be understood by the double Gaussian approximation, where the probability distribution of the Polyakov loop, an order parameter, is described by the summation of two Gaussian distributions centred at two values of the order parameter [36, 37]. This picture emerges as follows. One can transform the path-integration for the expectation value of an observable to an integration over the order parameter by inserting $1=\int d u_{1} \delta\left(u_{1}-\frac{1}{N} \operatorname{Tr} \exp [i \beta A]\right)$ and integrating out all the matrix fields. The resultant expectation value of an observable, $\mathcal{O}$, is written as

$$
\frac{1}{z} \int d u_{1} \overline{\mathcal{O}}\left(u_{1}\right) e^{-f\left(\beta ; u_{1}\right)}
$$

where $z=\int d u_{1} e^{-f\left(\beta ; u_{1}\right)}$ and

$$
\overline{\mathcal{O}}\left(u_{1}\right)=e^{f\left(\beta ; u_{1}\right)} \int[d X][d A] \mathcal{O} \delta\left(u_{1}-\frac{1}{N} \operatorname{Tr} \exp [i \beta A]\right) e^{-S[\beta ; X, A]} .
$$

Its exact computation has not been achieved yet even for the non-interacting gauged Gaussian model. However, there is speculation based on approximations by the LandauGinzburg model and gauge/gravity duality [5, 12, 18, 29]: within $0 \leq u_{1} \leq 1, f\left(\beta ; u_{1}\right)$ is expected to have one minimum around $u_{1}=0$ at low enough temperatures and around $u_{1} \geq 1 / 2$ at high enough temperatures but with two local minima around $u_{1}=0$ and $u_{1}=1 / 2$ near the transition temperature. Thus, during the transition, the probability weight $e^{-f\left(\beta ; u_{1}\right)}$ is described by two Gaussian distributions around the minima. This is a natural explanation of the observed two-level nature.

The fact that the two-level nature of the transition in this model only becomes apparent for rather large $N$ is not encouraging for numerical studies of more complicated models with fermions. However, the growth of the specific heat with $N^{2}$ was apparent for smaller $N$, as seen in figure 2, suggesting it is useful to monitor this observable whenever possible if the nature of the transition is in question. Such issues might be problematic in the class of supersymmetric models especially the BMN model where a rich phase structure is expected.

However, there is still room for discussion about the nature of the observed transition. As discussed in $[5,6]$ by using state-counting analysis, the Hagedorn behaviour is predicted in matrix models. In fact, we observe that the $N$-dependence of the Polyakov loop at lowtemperature phase is well fit by the prediction based on the Hagedorn behaviour [42], where the finite- $N$ effects are large even for very large $N$ in the transition region. Hence, there is little doubt that the low-temperature phase of the model has fluctuations characteristic of a Hagedorn transition. 
In future work we plan to investigate the model for more general values of $\mu$. Also we plan to return to the study of the D0-D4 Berkooz-Douglas model [39-41]. It would be natural to perform a similar study to the current one for the bosonic version where one can investigate the effect of the fundamental degrees of freedom on the system. This model becomes especially interesting for $N_{f}=2 N$ whose supersymmetric version is the dimensional reduction to time of the superconformally invariant four-dimensional model. An initial study of this model was performed in [9] and the exceptional behaviour of $N_{f}=2 N$ was noted.

\section{Acknowledgments}

The authors wish to acknowledge the Irish Centre for High-End Computing (ICHEC) for the provision of computational facilities and support (Projects dsphy009c, dsphy010c and dsphy012c). The support from Action MP1405 QSPACE of the COST foundation is gratefully acknowledged. Y. Asano is supported by the JSPS Research Fellowship for Young Scientists. S. Kováčik was supported by Irish Research Council funding. The authors would like to thank G. Bergner, M. Hanada, G. Ishiki, T. Morita and H. Watanabe for valuable discussions.

Open Access. This article is distributed under the terms of the Creative Commons Attribution License (CC-BY 4.0), which permits any use, distribution and reproduction in any medium, provided the original author(s) and source are credited.

\section{References}

[1] B. de Wit, J. Hoppe and H. Nicolai, On the Quantum Mechanics of Supermembranes, Nucl. Phys. B 305 (1988) 545 [INSPIRE].

[2] T. Banks, W. Fischler, S.H. Shenker and L. Susskind, $M$ theory as a matrix model: A Conjecture, Phys. Rev. D 55 (1997) 5112 [hep-th/9610043] [INSPIRE].

[3] D.E. Berenstein, J.M. Maldacena and H.S. Nastase, Strings in flat space and pp waves from $N=4$ superYang-Mills, JHEP 04 (2002) 013 [hep-th/0202021] [INSPIRE].

[4] N. Kim and J.-H. Park, Massive super Yang-Mills quantum mechanics: Classification and the relation to supermembrane, Nucl. Phys. B 759 (2006) 249 [hep-th/0607005] [INSPIRE].

[5] O. Aharony, J. Marsano, S. Minwalla, K. Papadodimas and M. Van Raamsdonk, The Hagedorn-deconfinement phase transition in weakly coupled large $N$ gauge theories, hep-th/0310285 [INSPIRE].

[6] K. Furuuchi, E. Schreiber and G.W. Semenoff, Five-brane thermodynamics from the matrix model, hep-th/0310286 [INSPIRE].

[7] G.W. Semenoff, Black holes and thermodynamic states of matrix models, in From fields to strings, vol. 3, M. Shifman et al. eds., pp. 2009-2034 (2005) [INSPIRE].

[8] O. Aharony, J. Marsano, S. Minwalla, K. Papadodimas, M. Van Raamsdonk and T. Wiseman, The Phase structure of low dimensional large $N$ gauge theories on Tori, JHEP 01 (2006) 140 [hep-th/0508077] [INSPIRE]. 
[9] Y. Asano, V.G. Filev, S. Kováčik and D. O'Connor, The non-perturbative phase diagram of the BMN matrix model, JHEP 07 (2018) 152 [arXiv: 1805.05314] [INSPIRE].

[10] R. Gregory and R. Laflamme, Black strings and p-branes are unstable, Phys. Rev. Lett. 70 (1993) 2837 [hep-th/9301052] [INSPIRE].

[11] R. Gregory and R. Laflamme, The Instability of charged black strings and p-branes, Nucl. Phys. B 428 (1994) 399 [hep-th/9404071] [INSPIRE].

[12] O. Aharony, J. Marsano, S. Minwalla and T. Wiseman, Black hole-black string phase transitions in thermal $1+1$ dimensional supersymmetric Yang-Mills theory on a circle, Class. Quant. Grav. 21 (2004) 5169 [hep-th/0406210] [INSPIRE].

[13] N. Kawahara, J. Nishimura and S. Takeuchi, Phase structure of matrix quantum mechanics at finite temperature, JHEP 10 (2007) 097 [arXiv:0706.3517] [INSPIRE].

[14] V.G. Filev and D. O'Connor, The BFSS model on the lattice, JHEP 05 (2016) 167 [arXiv: 1506.01366] [INSPIRE].

[15] T. Azuma, T. Morita and S. Takeuchi, Hagedorn Instability in Dimensionally Reduced Large- $N$ Gauge Theories as Gregory-Laflamme and Rayleigh-Plateau Instabilities, Phys. Rev. Lett. 113 (2014) 091603 [arXiv: 1403.7764] [INSPIRE].

[16] G. Mandal, M. Mahato and T. Morita, Phases of one dimensional large $N$ gauge theory in a 1/D expansion, JHEP 02 (2010) 034 [arXiv:0910.4526] [inSPIRE].

[17] G. Bergner, N. Bodendorfer, M. Hanada, E. Rinaldi, A. Schäfer and P. Vranas, Thermal phase transition in Yang-Mills matrix model, JHEP 01 (2020) 053 [arXiv:1909.04592] [INSPIRE].

[18] T. Morita and H. Yoshida, Critical Dimension and Negative Specific Heat in One-dimensional Large-N Reduced Models, Phys. Rev. D 101 (2020) 106010 [arXiv:2001.02109] [inSPIRE].

[19] M. Hanada, G. Ishiki and H. Watanabe, Partial Deconfinement, JHEP 03 (2019) 145 [Erratum ibid. 10 (2019) 029] [arXiv: 1812.05494] [INSPIRE].

[20] M. Hanada and J. Maltz, A proposal of the gauge theory description of the small Schwarzschild black hole in $A d S_{5} \times S^{5}$, JHEP 02 (2017) 012 [arXiv:1608.03276] [INSPIRE].

[21] D. Berenstein, Submatrix deconfinement and small black holes in AdS, JHEP 09 (2018) 054 [arXiv: 1806.05729] [INSPIRE].

[22] M. Hanada, A. Jevicki, C. Peng and N. Wintergerst, Anatomy of Deconfinement, JHEP 12 (2019) 167 [arXiv: 1909.09118] [INSPIRE].

[23] R. Emparan, R. Luna, M. Martínez, R. Suzuki and K. Tanabe, Phases and Stability of Non-Uniform Black Strings, JHEP 05 (2018) 104 [arXiv:1802.08191] [INSPIRE].

[24] Ó.J.C. Dias, J.E. Santos and B. Way, Localised and nonuniform thermal states of super-Yang-Mills on a circle, JHEP 06 (2017) 029 [arXiv: 1702.07718] [INSPIRE].

[25] B. Cardona and P. Figueras, Critical Kaluza-Klein black holes and black strings in D $=10$, JHEP 11 (2018) 120 [arXiv:1806.11129] [INSPIRE].

[26] M. Ammon, M. Kalisch and S. Moeckel, Notes on ten-dimensional localized black holes and deconfined states in two-dimensional SYM, JHEP 11 (2018) 090 [arXiv:1806.11174] [INSPIRE].

[27] E. Witten, Anti-de Sitter space, thermal phase transition and confinement in gauge theories, Adv. Theor. Math. Phys. 2 (1998) 505 [hep-th/9803131] [INSPIRE]. 
[28] Y. Asano and D. O'Connor, Wilson fermions in thermodynamics of Matrix theory, in preparation.

[29] S. Hadizadeh, B. Ramadanovic, G.W. Semenoff and D. Young, Free energy and phase transition of the matrix model on a plane-wave, Phys. Rev. D 71 (2005) 065016 [hep-th/0409318] [INSPIRE].

[30] S. Takeuchi, D-dependence of the gap between the critical temperatures in the one-dimensional gauge theories, Eur. Phys. J. C 79 (2019) 548 [arXiv:1712.09261] [INSPIRE].

[31] R. Delgadillo-Blando, D. O'Connor and B. Ydri, Geometry in Transition: A Model of Emergent Geometry, Phys. Rev. Lett. 100 (2008) 201601 [arXiv:0712.3011] [INSPIRE].

[32] R. Delgadillo-Blando, D. O'Connor and B. Ydri, Matrix Models, Gauge Theory and Emergent Geometry, arXiv:0806.0558 [INSPIRE].

[33] M.S. Costa, L. Greenspan, J. Penedones and J. Santos, Thermodynamics of the BMN matrix model at strong coupling, JHEP 03 (2015) 069 [arXiv:1411.5541] [INSPIRE].

[34] R. Delgadillo-Blando and D. O'Connor, Matrix geometries and Matrix Models, JHEP 11 (2012) 057 [arXiv: 1203.6901] [INSPIRE].

[35] M.E. Fisher and A.N. Berker, Scaling for first-order transitions in thermodynamic and finite systems, Phys. Rev. B 26 (1982) 2507 [InSPIRE].

[36] K. Binder and D.P. Landau, Finite-size scaling at first-order phase transitions, Phys. Rev. B 30 (1984) 1477 [INSPIRE].

[37] M.S.S. Challa, D.P. Landau and K. Binder, Finite size effects at temperature driven first order transitions, Phys. Rev. B 34 (1986) 1841 [INSPIRE].

[38] L. Glaser, D. O'Connor and S. Surya, Finite Size Scaling in $2 d$ Causal Set Quantum Gravity, Class. Quant. Grav. 35 (2018) 045006 [arXiv:1706.06432] [INSPIRE].

[39] V.G. Filev and D. O'Connor, A Computer Test of Holographic Flavour Dynamics, JHEP 05 (2016) 122 [arXiv: 1512.02536] [inSPIRE].

[40] Y. Asano, V.G. Filev, S. Kováčik and D. O'Connor, The flavoured BFSS model at high temperature, JHEP 01 (2017) 113 [arXiv:1605.05597] [INSPIRE].

[41] Y. Asano, V.G. Filev, S. Kováčik and D. O'Connor, A computer test of holographic avour dynamics. Part II, JHEP 03 (2018) 055 [arXiv: 1612.09281] [INSPIRE].

[42] S. Kováčik, D. O'Connor and Y. Asano, The nonperturbative phase diagram of the bosonic BMN matrix model, arXiv:2004.05820 [INSPIRE]. 